\title{
THE IDEAL STRUCTURE OF SEMIGROUPS OF TRANSFORMATIONS WITH RESTRICTED RANGE
}

\author{
SUZANA MENDES-GONÇALVES ${ }^{\otimes}$ and R. P. SULLIVAN
}

(Received 16 June 2010)

\begin{abstract}
Let $Y$ be a fixed nonempty subset of a set $X$ and let $T(X, Y)$ denote the semigroup of all total transformations from $X$ into $Y$. In 1975, Symons described the automorphisms of $T(X, Y)$. Three decades later, Nenthein, Youngkhong and Kemprasit determined its regular elements, and more recently Sanwong, Singha and Sullivan characterized all maximal and minimal congruences on $T(X, Y)$. In 2008, Sanwong and Sommanee determined the largest regular subsemigroup of $T(X, Y)$ when $|Y| \neq 1$ and $Y \neq X$; and using this, they described the Green's relations on $T(X, Y)$. Here, we use their work to describe the ideal structure of $T(X, Y)$. We also correct the proof of the corresponding result for a linear analogue of $T(X, Y)$.
\end{abstract}

2010 Mathematics subject classification: primary 20M20; secondary 15A04.

Keywords and phrases: transformation semigroups, ideals, maximal, reductive.

\section{Introduction}

Let $X$ be a nonempty set and let $T(X)$ denote the semigroup (under composition) of all total transformations of $X$. For each $\alpha$ in $T(X)$, we let $X \alpha=\operatorname{ran} \alpha$ denote the range of $\alpha$ and we define the rank of $\alpha$ to be $r(\alpha)=|\operatorname{ran} \alpha|$. If $\emptyset \neq Y \subseteq X$, we write

$$
T(X, Y)=\{\alpha \in T(X): X \alpha \subseteq Y\} .
$$

Clearly $T(X, Y)$ is a subsemigroup of $T(X)$, and if $Y=X$ then $T(X, Y)=T(X)$. Also, if $|Y|=1$ then $T(X, Y)$ contains exactly one element: the constant map with range $Y$. Hence, throughout the following, we assume that $Y$ is a proper subset of $X$ with at least two elements.

In [9], Symons described all the automorphisms of $T(X, Y)$. Several years later, its regular elements were characterized in [4]. Also, in [6], the authors determined the largest regular subsemigroup of $T(X, Y)$ and, using this, they described Green's relations on $T(X, Y)$. More recently, in [5], Sanwong et al. characterized all maximal and minimal congruences on $T(X, Y)$.

The authors acknowledge the support of the Portuguese 'Fundação para a Ciência e a Tecnologia' through its Multi-Year Funding Program for 'Centro de Matemática' at the University of Minho, Braga, Portugal.

(C) 2010 Australian Mathematical Publishing Association Inc. 0004-9727/2010 \$16.00 
In [8] Sullivan described Green's relations and ideals in a linear analogue of $T(X, Y)$. Namely, if $W$ is a nonzero proper subspace of a vector space $V$, we let $T(V, W)$ denote the semigroup (under composition) of all linear $\alpha: V \rightarrow V$ such that $V \alpha \subseteq W$. That is, we use the ' $V$ ' and ' $W$ ' in $T(V, W)$ to denote the fact that we are considering linear transformations. By [8, Corollary 12], $T(V, W)$ is rarely isomorphic to the semigroup $T(U)$ of all linear transformations of an arbitrary vector space $U$. In addition, whereas $T(V, W)$ always contains a zero element (namely, the map $V \rightarrow\{0\})$, the same is not true for $T(X, Y)$ if $|Y| \geq 2$. Hence, these two semigroups are not isomorphic and so they are worthy of study in their own right.

In Section 4, using the work in [6], we describe the ideal structure of $T(X, Y)$ and, as a consequence, we prove that this semigroup is almost never isomorphic to $T(Z)$ for any set $Z$. Also, in Section 5, we show how certain algebraic semigroups can be 'antiembedded' in some $T(X, Y)$. However, before we present these nonlinear results, we correct the proof of [8, Theorem 11] which describes all of the ideals of $T(V, W)$ : the argument we give for this in Section 3 then suggests how to derive the corresponding result for $T(X, Y)$.

In effect, this paper completes a project in which Green's relations and ideals are determined for semigroups which appear to be related but are almost never isomorphic or anti-isomorphic: namely, the semigroup $T(X, Y)$ and its linear analogue $T(V, W)$, as well as the semigroups

$$
\begin{gathered}
K(V, W)=\{\alpha \in T(V): W \subseteq \operatorname{ker} \alpha\}, \\
E(X, \sigma)=\left\{\alpha \in T(X): \sigma \subseteq \pi_{\alpha}\right\},
\end{gathered}
$$

where $\sigma$ is a fixed equivalence on $X$ and $\pi_{\alpha}=\alpha \circ \alpha^{-1}$ (see [3, 7]).

\section{Green's relations on $T(X, Y)$}

Throughout this paper, we write $\mathrm{id}_{A}$ for the identity transformation on a set $A$ and we let $A_{b}$ denote the constant mapping with domain $A$ and range $\{b\}$. We also write $A \cup \dot{\cup} B$ for the disjoint union of sets $A$ and $B$. In addition, we adopt the convention introduced by Clifford and Preston in [1, Vol. 2, p. 241]: that is, if $\alpha \in T(X)$ then we write

$$
\alpha=\left(\begin{array}{c}
A_{i} \\
x_{i}
\end{array}\right)
$$

and take as understood that the subscript $i$ belongs to some (unmentioned) index set $I$, that the abbreviation $\left\{x_{i}\right\}$ denotes $\left\{x_{i}: i \in I\right\}$, and that $\operatorname{ran} \alpha=\left\{x_{i}\right\}$ and $x_{i} \alpha^{-1}=A_{i}$.

Green's relations on $T(X)$ are well known: if $\alpha, \beta \in T(X)$, then $\alpha \mathcal{L} \beta$ if and only if $\operatorname{ran} \alpha=\operatorname{ran} \beta ; \alpha \mathcal{R} \beta$ if and only if $\pi_{\alpha}=\pi_{\beta} ; \alpha \mathcal{D} \beta$ if and only if $r(\alpha)=r(\beta)$; and $\mathcal{J}=\mathcal{D}$ (see [1, Vol. 1, Lemmas 2.5, 2.6 and 2.8 and Theorem 2.9]). In [6, Theorem 2.4], the authors determined the largest regular subsemigroup of $T(X, Y)$ when $X \neq Y$ and $|Y| \neq 1$ : the set $F$ given by

$$
F=\{\alpha \in T(X, Y): X \alpha \subseteq Y \alpha\}
$$


which is needed to describe Green's relations on $T(X, Y)$. This was done by Sanwong and Sommanee in [6, Theorems 3.2, 3.3, 3.7 and 3.9], and we quote their results for convenience.

Lemma 1. Let $\gamma \in F$ and $\beta \in T(X, Y)$. Then $\beta=\lambda \gamma$ for some $\lambda \in T(X, Y)$ if and only if $\operatorname{ran} \beta \subseteq \operatorname{ran} \gamma$. Consequently, if $\alpha, \beta \in T(X, Y)$, then $\alpha \mathcal{L} \beta$ in $T(X, Y)$ if and only if $\alpha=\beta$ or $(\operatorname{ran} \alpha=\operatorname{ran} \beta$ and $\alpha, \beta \in F)$.

Lemma 2. If $\alpha, \beta \in T(X, Y)$, then $\beta=\alpha \mu$ for some $\mu \in T(X, Y)$ if and only if $\pi_{\alpha} \subseteq \pi_{\beta}$. Consequently, $\alpha \mathcal{R} \beta$ in $T(X, Y)$ if and only if $\pi_{\alpha}=\pi_{\beta}$.

Lemma 3. If $\alpha, \beta \in T(X, Y)$, then $\alpha \mathcal{D} \beta$ in $T(X, Y)$ if and only if $\pi_{\alpha}=\pi_{\beta}$ or $(r(\alpha)=r(\beta)$ and $\alpha, \beta \in F)$.

LEMMA 4. If $\alpha, \beta \in T(X, Y)$, then $\beta=\lambda \alpha \mu$ for some $\lambda, \mu \in T(X, Y)$ if and only if $r(\beta) \leq|Y \alpha|$. Consequently, $\alpha \mathcal{J} \beta$ in $T(X, Y)$ if and only if $\pi_{\alpha}=\pi_{\beta}$ or $r(\alpha)=|Y \alpha|=$ $|Y \beta|=r(\beta)$.

By Hall's theorem [2, Proposition II.4.5], any regular subsemigroup of $T(X)$ inherits characterizations of its relations $\mathcal{L}$ and $\mathcal{R}$ from those on $T(X)$. Thus, by Lemmas 1 and 2, if $\alpha, \beta \in F$, then $\alpha \mathcal{L} \beta$ in $F$ if and only if $\operatorname{ran} \alpha=\operatorname{ran} \beta$, and $\alpha \mathcal{R} \beta$ in $F$ if and only if $\pi_{\alpha}=\pi_{\beta}$.

As observed in [6, Corollary 3.11], $\mathcal{J}=\mathcal{D}$ on $F$. In fact, the next result shows that if $\alpha, \beta \in F$, then $\alpha \mathcal{J} \beta$ in $F$ if and only if $r(\alpha)=r(\beta)$ : this is comparable with the $\mathcal{J}$-relation on $T(X)$.

LEMma 5. If $\alpha, \beta \in F$, then $\beta=\lambda \alpha \mu$ for some $\lambda, \mu \in F$ if and only if $r(\beta) \leq r(\alpha)$. Consequently, $\alpha \mathcal{J} \beta$ in $F$ if and only if $r(\alpha)=r(\beta)$.

ProOF. Suppose that $\beta=\lambda \alpha \mu$ for some $\lambda, \mu \in F$. By Lemma $4, r(\beta) \leq|Y \alpha|$. Since $\alpha \in F$, then $X \alpha \subseteq Y \alpha \subseteq X \alpha$, and so $|Y \alpha|=|X \alpha|=r(\alpha)$. Thus, $r(\beta) \leq r(\alpha)$. Conversely, suppose that the latter holds and let $\operatorname{ran} \beta=\left\{b_{i}\right\}$ and $\operatorname{ran} \alpha=\left\{a_{i}\right\} \dot{\cup}\left\{a_{j}\right\}$, where $\left\{b_{i}\right\}=Y \beta=X \beta \subseteq Y$ and $\left\{a_{i}\right\} \dot{\cup}\left\{a_{j}\right\}=Y \alpha=X \alpha \subseteq Y$. For each $i$, let $b_{i} \beta^{-1}=$ $B_{i}$ and $a_{i} \alpha^{-1}=A_{i}$, and choose $y_{i} \in A_{i} \cap Y$ (possible since $a_{i} \in Y \alpha$ ). Define $\lambda \in$ $T(X)$ by

$$
\lambda=\left(\begin{array}{c}
B_{i} \\
y_{i}
\end{array}\right) .
$$

Clearly, $X \lambda=\left\{y_{i}\right\} \subseteq Y$. Since $\left\{b_{i}\right\}=Y \beta$, it follows that $B_{i} \cap Y \neq \emptyset$ for every $i$. Therefore, $Y \lambda=\left\{y_{i}\right\}=X \lambda$, and hence $\lambda \in F$. Now fix $i_{0} \in I$ and let $Y \backslash X \alpha=\left\{a_{k}\right\}$ (note that this set may be empty). Write $\left\{a_{j}\right\} \dot{\cup}\left\{a_{k}\right\} \dot{\cup}(X \backslash Y)=C$ and define $\mu \in T(X)$ by

$$
\mu=\left(\begin{array}{cc}
a_{i} & C \\
b_{i} & b_{i_{0}}
\end{array}\right) .
$$

Then $X \mu=Y \mu=\left\{b_{i}\right\} \subseteq Y$, and so $\mu \in F$. Also $\beta=\lambda \alpha \mu$. 
Next we show that if $\alpha \mathcal{J} \beta$ in $F$ then $r(\alpha)=r(\beta)$ (the converse follows from the first part of this lemma). Suppose that $\beta=\lambda \alpha \mu$ and $\alpha=\lambda^{\prime} \beta \mu^{\prime}$ for some $\lambda, \lambda^{\prime}, \mu, \mu^{\prime} \in$ $F^{1}$. Then

$$
|X \beta|=|(X \lambda) \alpha \mu| \leq|(X \alpha) \mu| \leq|X \alpha|,
$$

even if $\lambda=1$ or $\mu=1$. Similarly, $|X \alpha| \leq\left|\left(X \lambda^{\prime}\right) \beta \mu^{\prime}\right| \leq|X \beta|$, and hence $r(\alpha)=$ $r(\beta)$.

Although the $\mathcal{R}$-relation on $T(X, Y)$ can be described just like the corresponding one on $T(X)$, the other Green's relations differ substantially from the corresponding ones on $T(X)$. In particular, from Lemma 4, we conclude that $\alpha \mathcal{J} \beta$ in $T(X, Y)$ implies that $r(\alpha)=r(\beta)$, but the converse does not hold when $X \neq Y$ and $|Y| \neq 1$. To see this, choose two distinct elements $y_{1}, y_{2}$ in $Y$ and write $Y=A \cup \dot{\cup} B$, with $y_{1} \in A$ and $y_{2} \in B$. Also, let $X \backslash Y=C$. Now define $\alpha, \beta \in T(X)$ by

$$
\alpha=\left(\begin{array}{cc}
A \dot{\cup} B & C \\
y_{1} & y_{2}
\end{array}\right), \quad \beta=\left(\begin{array}{cc}
A \dot{\cup} C & B \\
y_{2} & y_{1}
\end{array}\right) .
$$

Clearly, $\alpha, \beta \in T(X, Y)$ and $r(\alpha)=r(\beta)$, since $\operatorname{ran} \alpha=\operatorname{ran} \beta=\left\{y_{1}, y_{2}\right\} \subseteq Y$. On the other hand, $|Y \alpha| \neq|Y \beta|$ and $\pi_{\alpha} \neq \pi_{\beta}$, and this implies that $\alpha$ and $\beta$ are not $\mathcal{J}$-related in $T(X, Y)$.

In passing, we observe that in [6, Theorem 3.12], the authors proved that if $Y$ is finite, then $\mathcal{D}=\mathcal{J}$ on $T(X, Y)$, but the same does not hold in general (see [6, Example 3.10]).

\section{Ideals in $T(V, W)$}

Before determining all of the ideals in $T(X, Y)$, we correct the proof of the corresponding result for $T(V, W)$ in [8, Theorem 11]. The argument for that result appeals to [8, Lemma 10] where, using the notation of its proof, $\left\{w_{m}\right\} \dot{\cup}\left\{w_{n}\right\}$ is a linearly independent subset of $W$ and $u \in V \backslash W$, so $\left\{w_{m}\right\} \dot{\cup}\left\{u+w_{n}\right\}$ is linearly independent in $V$ and each $u+w_{n} \notin W$. However, it is asserted that $\operatorname{dim}(W \gamma)<$ $\operatorname{dim}(V \gamma)$ for some $\gamma \in T(V, W)$, which may be false. For example, $\left(u+w_{1}\right)-$ $\left(u+w_{2}\right) \in W$ if $1,2 \in N$ (see [8, p. 450]), and this may change the relative dimensions of $W \gamma$ and $V \gamma$. The result in [8, Theorem 11] is correct, but it requires a different lemma (recall that, as assumed in [8, p. 442], to avoid trivialities, $W$ is a nonzero proper subspace of $V$ ). In what follows, we use the notation of [8], but change it slightly to avoid any confusion with our notation in Section 4.

As in [8, p. 442], we let $Q=\{\alpha \in T(V, W): V \alpha \subseteq W \alpha\}$. By [8, Lemma 1], $Q$ is the largest regular subsemigroup of $T(V, W)$.

LEMMA 6. If $\beta \in Q$ and $r<\operatorname{dim}(W \beta)=s$, then there exists $\lambda \in T(V, W)$ such that $\lambda \beta \notin Q$ and $\operatorname{dim}(W \lambda \beta)=r$.

PROOF. If $\beta \in Q$ and $\operatorname{dim}(W \beta)=s \geq r^{\prime}$, we can write

$$
\beta=\left(\begin{array}{cc}
u_{p} & w_{j} \\
0 & w_{j}^{\prime}
\end{array}\right)
$$


where $|J|=s$. Choose $K \dot{U}\{1\} \subseteq J$ with $|K|=r$, let $u \in V \backslash W$, write $V=\left\langle v_{\ell}\right\rangle \oplus$ $\langle u\rangle \oplus\left\langle w_{k}\right\rangle$ where $W \subseteq\left\langle v_{\ell}\right\rangle \oplus\left\langle w_{k}\right\rangle$, and define $\lambda \in T(V, W)$ by

$$
\lambda=\left(\begin{array}{ccc}
v_{\ell} & u & w_{k} \\
0 & w_{1} & w_{k}
\end{array}\right) .
$$

Then $W \lambda \beta=\left\langle w_{k}^{\prime}\right\rangle \neq\left\langle w_{1}^{\prime}\right\rangle \oplus\left\langle w_{k}^{\prime}\right\rangle=V \lambda \beta$, so $\lambda \beta \notin Q$ and $\operatorname{dim}(W \lambda \beta)=r$.

We now prove [8, Theorem 11]: in essence, the only difference between what follows and the argument for [8, Theorem 11] lies in the choice of the subset $\Sigma$ of the ideal $\mathbb{I}$ in $T(V, W)$. For convenience, we recall some notation in [8, p. 448]: namely, for each $1 \leq r \leq \operatorname{dim} W, T_{r}$ denotes the set $\{\alpha \in T(V, W): r(\alpha)<r\}$, and if $\Sigma$ is a nonempty subset of $T(V, W)$, then

$$
\begin{gathered}
r(\Sigma)=\min \{r: r>\operatorname{dim}(W \alpha) \text { for all } \alpha \in \Sigma\}, \\
K(\Sigma)=\{\beta \in T(V, W): \operatorname{ker} \beta \supseteq \operatorname{ker} \alpha \text { for some } \alpha \in \Sigma\} .
\end{gathered}
$$

THEOREM 7. The ideals of $T(V, W)$ are precisely the sets $T_{r} \cup K(\Sigma)$ and $T_{r^{\prime}} \cup$ $K(\Sigma)$, where $r=r(\Sigma)$ and $\Sigma$ is a nonempty subset of $T(V, W)$.

PROOF. Let $\mathbb{I}$ be an ideal of $T(V, W)$. If $\mathbb{I}=\{0\}$, we let $\Sigma=\mathbb{I}$, so $r(\Sigma)=1$, $T_{1}=\{0\}$; and, if $\beta \in K(\{0\})$ then $\operatorname{ker} \beta=V$, so $\beta=0$ and thus $K(\{0\})=\{0\}$. That is, $\{0\}=T_{1} \cup K(\{0\})$.

Suppose $\alpha \in \mathbb{I}$ is nonzero and write

$$
\alpha=\left(\begin{array}{ccc}
u_{p} & w_{j} & v_{k} \\
0 & w_{j}^{\prime} & w_{k}
\end{array}\right)
$$

where $W \subseteq\left\langle u_{p}\right\rangle \oplus\left\langle w_{j}\right\rangle$ and $W \cap\left\langle v_{k}\right\rangle=\{0\}$. If $J=\emptyset$, then $K \neq \emptyset$ and $W \alpha=\{0\} \neq$ $\left\langle w_{k}\right\rangle=V \alpha$, so $\alpha \in \mathbb{I} \backslash Q$. On the other hand, if $J \neq \emptyset$, choose $1 \in J$ and $u \in V \backslash W$, write $V=\langle u\rangle \oplus\left\langle v_{m}\right\rangle$ where $W \subseteq\left\langle v_{m}\right\rangle$, and let

$$
\lambda=\left(\begin{array}{cc}
v_{m} & u \\
0 & w_{1}
\end{array}\right) .
$$

Then $W \lambda \alpha=\{0\} \neq\left\langle w_{1}^{\prime}\right\rangle=V \lambda \alpha$, so $\lambda \alpha \in \mathbb{I}$ and $\lambda \alpha \notin Q$. That is, in each case, if $\Sigma=\mathbb{I} \backslash Q$ then $\Sigma \neq \varnothing$ and we assert that $\mathbb{I}$ equals $T_{r} \cup K(\Sigma)$ or $T_{r^{\prime}} \cup K(\Sigma)$, where $r=r(\Sigma)$.

First suppose that $\operatorname{dim}(W \beta)<r$ for all $\beta \in \mathbb{I}$. In this case, suppose that $\beta \in \mathbb{I}$. Now, if $r(\beta)<r$, then $\beta \in T_{r}$ and, if $\operatorname{dim}(W \beta)<r \leq r(\beta)$, then $W \beta \neq V \beta$, so $\beta \in \Sigma$ and hence $\beta \in K(\Sigma)$. Thus, in this case, $\mathbb{I} \subseteq T_{r} \cup K(\Sigma)$. Conversely, suppose that $\beta \in T_{r}$. If $\operatorname{dim}(W \alpha)<r(\beta)<r$ for all $\alpha \in \Sigma$, we contradict the choice of $r=r(\Sigma)$. Therefore, $r(\beta) \leq \operatorname{dim}(W \alpha)$ for some $\alpha \in \Sigma \subseteq \mathbb{I}$, and hence $\beta \in \mathbb{I}$ by [8, Lemma 4]. Clearly, $K(\Sigma) \subseteq \mathbb{I}$ by [8, Lemma 3], so we conclude that $\mathbb{I}=T_{r} \cup K(\Sigma)$.

Next suppose that $r \leq \operatorname{dim}(W \pi)$ for some $\pi \in \mathbb{I}$. In this case, if $W \pi \neq V \pi$, then $\pi \in \Sigma$ and we contradict the choice of $r$. Hence $W \pi=V \pi$ and thus $\pi \in Q$, where $r(\pi)=s \geq r$. Now, if $s \geq r^{\prime}$, then Lemma 6 says that there exists $\lambda \in T(V, W)$ such that $\lambda \pi \in \mathbb{I} \backslash Q=\Sigma$ and $\operatorname{dim}(W \lambda \pi)=r$, which contradicts the choice of $r$. 
Hence, in this case, $r=s$ and thus $\pi \in T_{r^{\prime}}$. Clearly this conclusion holds for any $\beta \in \mathbb{I}$ such that $r \leq \operatorname{dim}(W \beta)$. On the other hand, if $\beta \in \mathbb{I}$ and $\operatorname{dim}(W \beta)<r$, then we have already seen that $\beta \in T_{r} \cup K(\Sigma)$. So, in this case, $\mathbb{I} \subseteq T_{r^{\prime}} \cup K(\Sigma)$. Conversely, if $\beta \in T_{r^{\prime}}$ then $r(\beta) \leq r=\operatorname{dim}(W \pi)$ for the same $\pi$ as before, so $\beta \in \mathbb{I}$ by [8, Lemma 4]. Like before, $K(\Sigma) \subseteq \mathbb{I}$, and we now conclude that $\mathbb{I}=T_{r^{\prime}} \cup K(\Sigma)$.

\section{Ideals in $T(X, Y)$}

As in Section 3, for each cardinal $r$, we let $r^{\prime}$ denote the successor of $r$. It is well known that the ideals of $T(X)$ are precisely the sets $\{\alpha \in T(X): r(\alpha)<r\}$, where $1<r \leq|X|^{\prime}$, and hence they form a chain under containment. The same is true for the ideals in $F$, as we now show.

THEOREM 8. The ideals in $F$ are exactly the sets

$$
F_{r}=\{\alpha \in F: r(\alpha)<r\},
$$

where $1<r \leq|Y|^{\prime}$. Moreover, $F_{r}$ is a principal ideal of $F$ if and only if $r$ is a successor cardinal.

PROOF. It is easy to see that $F_{r}$ is nonempty. For, given $y \in Y, r\left(X_{y}\right)=1<r$ and so $X_{y} \in F_{r}$. Now let $\alpha \in F_{r}$ and $\beta \in F$. Then $\alpha \beta, \beta \alpha \in F$ and

$$
r(\alpha \beta)=|X \alpha \beta| \leq|X \alpha|=r(\alpha)<r .
$$

Also $X \beta \alpha \subseteq X \alpha$, and so $r(\beta \alpha) \leq r(\alpha)<r$. Therefore $\alpha \beta, \beta \alpha \in F_{r}$, and hence $F_{r}$ is an ideal of $F$. Conversely, let $\mathbb{I}$ be an ideal of $F$ and let $r$ be the least cardinal greater than $r(\alpha)$ for every $\alpha \in \mathbb{I}$ (this is possible since the cardinals are well ordered). Then $\mathbb{I} \subseteq F_{r}$. To see that $F_{r} \subseteq \mathbb{I}$, let $\beta \in F_{r}$. Then there exists $\alpha \in \mathbb{I}$ such that $r(\beta) \leq r(\alpha)$; otherwise, $r(\alpha)<r(\beta)<r$ for every $\alpha \in \mathbb{I}$, and this contradicts our choice of $r$. By Lemma $5, r(\beta) \leq r(\alpha)$ implies that $\beta=\lambda \alpha \mu$ for some $\lambda, \mu \in F$. Since $\mathbb{I}$ is an ideal of $F, \beta \in \mathbb{I}$, and so $F_{r}=\mathbb{I}$.

Next we determine all the principal ideals of $F$. To do this, let $r$ be a successor cardinal, say $r=s^{\prime}$, and choose $\alpha \in F_{r}$ with $r(\alpha)=s$. If $r(\beta)>s$ for some $\beta \in F_{r}$, then $r(\beta) \geq s^{\prime}=r$, a contradiction. Thus, for every $\beta \in F_{r}, r(\beta) \leq s=r(\alpha)$ and, by Lemma 5, $\beta \in J(\alpha)$, the principal ideal of $F$ generated by $\alpha$. Hence, $F_{r} \subseteq J(\alpha)$. Since the reverse inclusion also holds, $F_{r}$ is principal. Conversely, suppose that $F_{r}=J(\alpha)$ for some $\alpha \in F_{r}$. Let $r(\alpha)=s$ and assume that $s<t<r$ for some cardinal $t$. Clearly, $t=r(\gamma)$ for some $\gamma \in F$ (since $t<r \leq|Y|^{\prime}$ ). By Lemma 5, $J(\alpha) \subseteq J(\gamma) \subseteq F_{r}$, contradicting our supposition. In other words, $r$ is the least cardinal greater than $s$, and so $r=s^{\prime}$.

We proceed to describe the ideals of $T(X, Y)$. To do this, let $1<r \leq|Y|^{\prime}$ and write

$$
T_{r}=\{\alpha \in T(X, Y): r(\alpha)<r\} .
$$

Let $\alpha \in T_{r}$ and $\beta \in T(X, Y)$. Then $X \beta \alpha \subseteq X \alpha$, and so $r(\beta \alpha) \leq r(\alpha)<r$. Also $r(\alpha \beta)=|X \alpha \beta| \leq|X \alpha|=r(\alpha)<r$. Therefore, $T_{r}$ is an ideal of $T(X, Y)$. 
Now let $\mathfrak{S}$ be a nonempty subset of $T(X, Y)$ and let

$$
\begin{gathered}
r(\mathfrak{S})=\min \{r:|Y \alpha|<r \text { for every } \alpha \in \mathfrak{S}\}, \\
\Pi(\mathfrak{S})=\left\{\beta \in T(X, Y): \pi_{\alpha} \subseteq \pi_{\beta} \text { for some } \alpha \in \mathfrak{S}\right\} .
\end{gathered}
$$

LEMMA 9. For each nonempty subset $\mathfrak{S}$ of $T(X, Y), T_{r(\mathfrak{S})} \cup \Pi(\mathfrak{S})$ and $T_{r(\mathfrak{S})^{\prime}} \cup$ $\Pi(\mathfrak{S})$ are ideals of $T(X, Y)$.

Proof. Given $\beta, \mu \in T(X, Y), \pi_{\beta} \subseteq \pi_{\beta \mu}$. Thus, $\Pi(\mathfrak{S})$ is a right ideal of $T(X, Y)$. Now, let $\lambda \in T(X, Y)$ and $\beta \in \Pi(\mathfrak{S})$. Then $\pi_{\alpha} \subseteq \pi_{\beta}$ for some $\alpha \in \mathfrak{S}$ and, by Lemma $2, \beta=\alpha \mu$ for some $\mu \in T(X, Y)$. Therefore, since $X \lambda \subseteq Y$,

$$
r(\lambda \beta)=|X \lambda \beta| \leq|Y \beta|=|Y \alpha \mu| \leq|Y \alpha|<r(\mathfrak{S}) .
$$

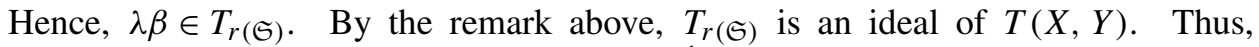
given $\beta \in T_{r(\mathfrak{S})} \cup \Pi(\mathfrak{S})$ and $\lambda, \mu \in T(X, Y)^{1}$, we have $\lambda \beta \mu \in T_{r(\mathfrak{S})} \cup \Pi(\mathfrak{S})$, and so $T_{r(\mathfrak{S})} \cup \Pi(\mathfrak{S})$ is an ideal of $T(X, Y)$. Since $T_{r(\mathfrak{S})^{\prime}}$ is an ideal of $T(X, Y)$ and $T_{r(\mathfrak{S})} \subseteq T_{r(\mathfrak{S})^{\prime}}$, it follows that $T_{r(\mathfrak{S})^{\prime}} \cup \Pi(\mathfrak{S})$ is also an ideal of $T(X, Y)$.

Next we show that the above ideals are the only ones in $T(X, Y)$. Although the following argument is similar to the one given for $T(V, W)$ in Section 3, we provide most of the details in this nonlinear context. As before, we start with a technical result.

LEMMA 10. If $\beta \in F$ and $r<|Y \beta|=s$, then there exists $\lambda \in T(X, Y)$ such that $\lambda \beta \notin F$ and $|Y \lambda \beta|=r$.

PROOF. If $\beta \in F$ and $|Y \beta|=s \geq r^{\prime}$, we can write

$$
\beta=\left(\begin{array}{c}
A_{j} \\
y_{j}^{\prime}
\end{array}\right)
$$

where $|J|=s$ and $Y \cap A_{j} \neq \emptyset$ for each $j$. Choose $K \dot{\cup}\{1\} \subseteq J$ with $|K|=r$, and let $y_{i} \in Y \cap A_{i}$ for each $i \in K \cup\{1\}$. Also, choose $2 \in K$ and write $L=K \backslash\{2\}$ (which may be empty). Finally, choose $u \in X \backslash Y$, let $B=X \backslash\left[\{u\} \cup\left\{y_{\ell}\right\}\right]$ and define $\lambda \in T(X, Y)$ by

$$
\lambda=\left(\begin{array}{ccc}
B & u & y_{\ell} \\
y_{2} & y_{1} & y_{\ell}
\end{array}\right) .
$$

Then $Y \lambda \beta=\left\{y_{2}^{\prime}\right\} \dot{\cup}\left\{y_{\ell}^{\prime}\right\} \neq X \lambda \beta$, so $\lambda \beta \notin F$ and $|Y \lambda \beta|=r$.

Recall that, as stated in Section $1, Y$ is a proper subset of $X$ with at least two elements. We let $C(Y)$ denote the set of all constants in $T(X, Y)$ and observe that this is the smallest ideal of $T(X, Y)$.

THEOREM 11. The ideals of $T(X, Y)$ are precisely the sets $T_{r} \cup \Pi(\mathfrak{S})$ and $T_{r^{\prime}} \cup$ $\Pi(\mathfrak{S})$, where $r=r(\mathfrak{S})$ and $\mathfrak{S}$ is a nonempty subset of $T(X, Y)$.

Proof. Let $\mathbb{I}$ be an ideal of $T(X, Y)$. If $\mathbb{I}=C(Y)$, we let $\mathfrak{S}=\mathbb{I}$, so $r(\mathfrak{S})=2$ and $T_{2}=C(Y)$; and, if $\beta \in \Pi(\mathfrak{S})$, then $\beta$ is constant and thus $\Pi(\mathfrak{S})=\mathfrak{S}$. That is, $C(Y)=T_{2} \cup \Pi(\mathfrak{S})$, where $\mathfrak{S}=C(Y)$. 
Suppose that $\alpha \in \mathbb{I}$ is nonconstant and write

$$
\alpha=\left(\begin{array}{cc}
A_{j} & A_{k} \\
y_{j}^{\prime} & y_{k}^{\prime}
\end{array}\right)
$$

where $Y \cap A_{j} \neq \emptyset$ for each $j$ and $Y \cap \bigcup A_{k}=\emptyset$. If $K \neq \emptyset$ then $Y \alpha=\left\{y_{j}^{\prime}\right\} \neq X \alpha$, so $\alpha \notin F$. On the other hand, if $K=\emptyset$ then $|J| \geq 2$. Now choose $1,2 \in J$ and $y_{i} \in A_{i} \cap Y$ for $i=1,2$, let $u \in X \backslash Y$ and define $\lambda \in T(X, Y)$ by

$$
\lambda=\left(\begin{array}{cc}
u & X \backslash\{u\} \\
y_{1} & y_{2}
\end{array}\right) .
$$

Then $Y \lambda \alpha=\left\{y_{2}^{\prime}\right\} \neq\left\{y_{1}^{\prime}, y_{2}^{\prime}\right\}=X \lambda \alpha$, so $\lambda \alpha \in \mathbb{I}$ and $\lambda \alpha \notin F$. That is, in each case, if $\mathfrak{S}=\mathbb{I} \backslash F$ then $\mathfrak{S} \neq \varnothing$ and we assert that $\mathbb{I}$ equals $T_{r} \cup \Pi(\mathfrak{S})$ or $T_{r^{\prime}} \cup \Pi(\mathfrak{S})$, where $r=r(\mathfrak{S})$.

First suppose that $|Y \beta|<r$ for all $\beta \in \mathbb{I}$. In this case, suppose that $\beta \in \mathbb{I}$. Now, if $r(\beta)<r$, then $\beta \in T_{r}$ and, if $|Y \beta|<r \leq r(\beta)$, then $Y \beta \neq X \beta$, so $\beta \in \mathfrak{S}$ and hence $\beta \in \Pi(\mathfrak{S})$. Thus, in this case, $\mathbb{I} \subseteq T_{r} \cup \Pi(\mathfrak{S})$. Conversely, suppose that $\beta \in T_{r}$. Then, as in the linear case, $r(\beta) \leq|Y \alpha|$ for some $\alpha \in \mathfrak{S} \subseteq \mathbb{I}$, and hence $\beta \in \mathbb{I}$ by Lemma 4 . Clearly, $\Pi(\mathfrak{S}) \subseteq \mathbb{I}$ by Lemma 2 , so we conclude that $\mathbb{I}=T_{r} \cup \Pi(\mathfrak{S})$.

Next suppose that $r \leq|Y \gamma|$ for some $\gamma \in \mathbb{I}$. In this case, if $Y \gamma \neq X \gamma$, then $\gamma \in \mathfrak{S}$ and we contradict the choice of $r$. Hence $Y \gamma=X \gamma$ and thus $\gamma \in F$, where $r(\gamma)=s \geq r$. Now, if $s \geq r^{\prime}$, then Lemma 10 says that there exists $\lambda \in T(X, Y)$ such that $\lambda \gamma \in \mathbb{I} \backslash F=\mathfrak{S}$ and $|Y \lambda \gamma|=r$, which contradicts the choice of $r$. Hence, in this case, $r=s$ and $\gamma \in T_{r^{\prime}}$. The rest of the proof proceeds in the same way as for Theorem 7, so we omit the details.

Corollary 12. If $|Y| \geq 3$, then $T(X, Y)$ is not isomorphic to $T(Z)$ for any set $Z$.

Proof. Suppose that $|Y| \geq 3$, write $Y$ as a disjoint union of three sets, say $A \dot{\cup} B \dot{\cup} C$, and let $y_{1}, y_{2}, y_{3} \in Y$ be distinct. By our assumption, $X \backslash Y \neq \emptyset$. Define $\alpha_{1}, \alpha_{2} \in$ $T(X, Y)$ by

$$
\alpha_{1}=\left(\begin{array}{ccc}
A \dot{\cup} B & C & X \backslash Y \\
y_{1} & y_{2} & y_{3}
\end{array}\right), \quad \alpha_{2}=\left(\begin{array}{ccc}
A & B \dot{\cup} C & X \backslash Y \\
y_{1} & y_{2} & y_{3}
\end{array}\right) .
$$

Clearly, $\left|Y \alpha_{1}\right|=2<3=\left|X \alpha_{1}\right|$ and so, if $\mathfrak{S}_{1}=\left\{\alpha_{1}\right\}$, then $r\left(\mathfrak{S}_{1}\right)=3$ and $\alpha_{1} \in T_{3} \cup$ $\Pi\left(\mathfrak{S}_{1}\right)$ and this is an ideal of $T(X, Y)$ by Lemma 9. Likewise, if $\mathfrak{S}_{2}=\left\{\alpha_{2}\right\}$ then $T_{3} \cup \Pi\left(\mathfrak{S}_{2}\right)$ is an ideal of $T(X, Y)$ and $\alpha_{2} \in T_{3} \cup \Pi\left(\mathfrak{S}_{2}\right)$. Now, $\alpha_{1} \notin T_{3} \cup \Pi\left(\mathfrak{S}_{2}\right)$ since $r\left(\alpha_{1}\right)=3$ and $\pi_{\alpha_{2}} \nsubseteq \pi_{\alpha_{1}}$, so $T_{3} \cup \Pi\left(\mathfrak{S}_{1}\right) \nsubseteq T_{3} \cup \Pi\left(\mathfrak{S}_{2}\right)$. Similarly, $r\left(\alpha_{2}\right)=3$ and $\pi_{\alpha_{1}} \nsubseteq \pi_{\alpha_{2}}$ imply $\alpha_{2} \notin T_{3} \cup \Pi\left(\mathfrak{S}_{1}\right)$, and hence $T_{3} \cup \Pi\left(\mathfrak{S}_{2}\right) \nsubseteq T_{3} \cup \Pi\left(\mathfrak{S}_{1}\right)$. In other words, we have shown that, if $|Y| \geq 3$, then $T(X, Y)$ contains two ideals which are not comparable under containment, and so it cannot be isomorphic to $T(Z)$ for any set $Z$. 
It is obvious that, if $|X| \geq 2$, then the largest proper ideal of $T(X)$ is $\{\alpha \in T(X)$ : $r(\alpha)<|X|\}$. However, to determine the maximal ideals in $T(X, Y)$, we need a technical lemma, which we motivate by observing that, for each $\alpha \in T(X, Y),|Y \alpha| \leq$ $|X \alpha| \leq|Y|$.

LEMMA 13. No proper ideal of $T(X, Y)$ contains any element $\gamma$ with $|Y \gamma|=|X \gamma|=$ $|Y|$.

PROOF. Let $\mathbb{J}$ be an ideal of $T(X, Y)$ and suppose that there exists $\gamma \in \mathbb{J}$ such that $|Y \gamma|=|X \gamma|=|Y|$. Given $\beta \in T(X, Y)$, we have $\operatorname{ran} \beta \subseteq Y$, and so $r(\beta) \leq|Y|=$ $|Y \gamma|$. By Lemma $4, \beta=\lambda \gamma \mu$ for some $\lambda, \mu \in T(X, Y)$, and so $\beta \in \mathbb{J}$. Therefore, $\mathbb{J}=T(X, Y)$.

THEOREM 14. If $|Y|=p \geq 2$, then the largest proper ideal of $T(X, Y)$ is the set $T_{p} \cup \mathfrak{S}$, where $\mathfrak{S}=\{\alpha \in T(X, Y):|Y \alpha|<|X \alpha|=p\}$ (which may be empty).

PROOF. First suppose that $\mathfrak{S}=\emptyset$. By the remark before Lemma $9, T_{p}$ is an ideal of $T(X, Y)$. Clearly, it is a proper ideal and, by Lemma 13, every proper ideal of $T(X, Y)$ is contained in $T_{p}$. Hence, in this case, $T_{p}$ is the largest proper ideal of $T(X, Y)$.

If $\mathfrak{S} \neq \emptyset$, then let $\alpha \in \mathfrak{S}$ and write $Y \alpha=\left\{a_{j}\right\}$. Since $|Y \alpha|<p=|X \alpha|$, we can write $X \alpha=\left\{a_{j}\right\} \dot{\cup}\left\{a_{i}\right\}$ for some subset $\left\{a_{i}\right\}$ of $Y$, where $|J|+|I|=p$. Clearly, $\left\{a_{i}\right\}=X \alpha \backslash Y \alpha \subseteq(X \backslash Y) \alpha$, and so $|X \backslash Y| \geq|I|$.

If $p$ is infinite, then $|X \backslash Y| \geq|I|=p=|Y|$ and so, for every cardinal $q$ such that $q<p$, we can write $Y=\left\{y_{m}\right\} \dot{\cup}\left\{y_{n}\right\}$ and $X \backslash Y=\left\{x_{n}\right\} \dot{\cup}\left\{x_{\ell}\right\}$, where $|M|=q$, $|N|=p$ and $|L|=|X \backslash Y|$. Choose $1 \in M$ and define $\beta \in T(X, Y)$ by

$$
\beta=\left(\begin{array}{cccc}
y_{m} & \left\{y_{n}\right\} & x_{n} & \left\{x_{\ell}\right\} \\
y_{m} & y_{1} & y_{n} & y_{1}
\end{array}\right) .
$$

Since $Y \beta=\left\{y_{m}\right\}$ and $X \beta=\left\{y_{m}\right\} \dot{\cup}\left\{y_{n}\right\}=Y$, it follows that $|Y \beta|=q$ and $\beta \in \mathfrak{S}$. That is, for each cardinal $q<p$, there exists $\beta \in \mathfrak{S}$ with $|Y \beta|=q$ and $\operatorname{so} r(\mathfrak{S})=p$.

Now suppose that $p \geq 2$ is finite and write $Y=\left\{y_{1}, \ldots, y_{p-1}, y_{p}\right\}$. Let $X \backslash Y=$ $\left\{x_{k}\right\}$ (nonempty since we assume $Y \subsetneq X$ ) and define $\beta \in T(X, Y)$ by

$$
\beta=\left(\begin{array}{ccccc}
y_{1} & \ldots & y_{p-1} & y_{p} & \left\{x_{k}\right\} \\
y_{1} & \ldots & y_{p-1} & y_{1} & y_{p}
\end{array}\right) .
$$

Clearly, $p-1=|Y \alpha|<|X \alpha|=p$, and so $r(\mathfrak{S})=p$.

By Lemma 9, $T_{p} \cup \Pi(\mathfrak{S})$ is an ideal of $T(X, Y)$. It is not difficult to see that $T_{p} \cup \Pi(\mathfrak{S})=T_{p} \cup \mathfrak{S}$. For example, clearly, $T_{p} \cup \mathfrak{S} \subseteq T_{p} \cup \Pi(\mathfrak{S})$. Given $\beta \in \Pi(\mathfrak{S})$, then $\pi_{\alpha} \subseteq \pi_{\beta}$ for some $\alpha \in \mathfrak{S}$. But this implies that $p>|Y \alpha| \geq|Y \beta|$. If $r(\beta)<p$, then $\beta \in T_{p}$. If not, then $\beta \in \mathfrak{S}$, and the equality follows. Also, if $\mathbb{J}$ is a proper ideal of $T(X, Y)$ then, by Lemma 13, $\mathbb{J} \subseteq T(X, Y) \backslash\{\alpha \in T(X, Y):|X \alpha|=|Y \alpha|=p\}$ : that is, $\mathbb{J} \subseteq T_{p} \cup \mathfrak{S}$ and this is the largest proper ideal of $T(X, Y)$. 
EXAMPLE 15. As in the proof of Theorem 14, it is easy to see that if $Y$ is finite, then $\mathfrak{S}$ is nonempty. Now suppose $|Y|=p \geq \aleph_{0}$ and $|X \backslash Y|<p$. Then $|X|=p$. Clearly, there exists $\alpha \in T(X, Y)$ such that $|X \alpha|=p$. For example, write $Y=\left\{y_{j}\right\}$ and $X=\left\{x_{j}\right\}$ with $|J|=p$, and define $\alpha \in T(X, Y)$ by

$$
\alpha=\left(\begin{array}{l}
x_{j} \\
y_{j}
\end{array}\right) .
$$

But, given $\beta \in T(X, Y)$ with $|X \beta|=p$, we know that $|Y \beta|=p$ (since $|(X \backslash Y) \beta| \leq$ $|X \backslash Y|<p)$, and so $\mathfrak{S}=\emptyset$ in this case.

\section{An embedding problem}

It is well known that any semigroup $S$ can be embedded in $T\left(S^{1}\right)$, where $S^{1}$ equals $S$ with an identity adjoined. This is achieved via the mapping $\rho: S \rightarrow T\left(S^{1}\right), a \rightarrow \rho_{a}$, where $\rho_{a}: S^{1} \rightarrow S^{1}, x \rightarrow x a$, for each $a \in S$. However, if we want $\rho$ to embed some $S$ into $T\left(S^{1}, Y\right)$ for some proper subset $Y$ of $S^{1}$, then we must have $S a \cup\{a\}=\operatorname{ran} \rho_{a} \subseteq$ $Y$ for all $a \in S$, and hence $Y=S$. On the other hand, if we do not add an identity to $S$, then we need $S$ to be 'cancellative' in some way: compare the embedding of a right cancellative semigroup $S$ into the semigroup of all injective transformations of $S$ in [1, Vol. 1, Lemma 1.0].

If $|Y| \geq 3$, then $T=T(X, Y)$ is right reductive (see [1, Vol. 1, p. 9]). In fact, it is $\mathfrak{S}$-right-reductive for some nonempty subset $\mathfrak{S}$ of $T$ : that is, if $\alpha \gamma=\beta \gamma$ for all $\gamma \in \mathfrak{S}$, then $\alpha=\beta$. For example, let $\mathfrak{S}_{3}$ denote the set of all $\gamma \in T$ with the form

$$
\gamma=\left(\begin{array}{ccc}
A & B & C \\
y_{1} & y_{2} & y_{3}
\end{array}\right)
$$

where precisely one of $A, B$ and $C$ contains no element of $Y$. Suppose that $\alpha, \beta \in T$ and $\alpha \gamma=\beta \gamma$ for all $\gamma \in \mathfrak{S}_{3}$, and assume that $x \alpha=y_{1} \neq y_{2}=x \beta$ for some $x \in X$. Now, since $|Y| \geq 3$ and there exists $u \in X \backslash Y$, we can write $X=A \dot{\cup}\left\{y_{2}\right\} \dot{\cup}\{u\}$ and let

$$
\gamma=\left(\begin{array}{ccc}
A & y_{2} & u \\
y_{1} & y_{2} & y_{3}
\end{array}\right) \in \mathfrak{S}_{3} .
$$

Then $x \alpha \gamma=y_{1}$ and $x \beta \gamma=y_{2}$, contradicting the supposition. That is, $x \alpha=x \beta$ for all $x \in X$, and thus $\alpha=\beta$.

Next recall that $T_{3}=\{\alpha \in T: r(\alpha)<3\}$ is an ideal of $T$, and observe that $\mathfrak{S}_{3}^{2} \subseteq T_{3}$. In fact, if we write an arbitrary $\alpha \in T$ as

$$
\alpha=\left(\begin{array}{ll}
A_{j} & A_{k} \\
y_{j} & y_{k}
\end{array}\right)
$$

where $Y \cap A_{j} \neq \emptyset$ for each $j$ and $Y \cap \bigcup A_{k}=\emptyset$, then it can be seen that $r(\alpha \gamma) \leq 2$ for each $\gamma \in \mathfrak{S}_{3}$. That is, for each $\alpha \in T, \alpha \mathfrak{S}_{3} \subseteq T_{3}$. Consequently, if $L=\mathfrak{S}_{3} \cup T_{3}$, then $L$ is a left ideal of $T(X, Y)$ and $\alpha L \subseteq T_{3} \subsetneq L$ for all $\alpha \in T$. 
With the above in mind, we say that, if $M, N$ are semigroups, then $\theta: M \rightarrow N$ is an anti-embedding if $\theta$ is injective and $(x y) \theta=(y \theta)(x \theta)$ for all $x, y \in M$. We now modify the regular anti-representation of a semigroup (see [1, Vol. 1, p. 9]) to antiembed certain semigroups into $T(X, Y)$ for some sets $X$ and $Y$.

THEOREM 16. Suppose $K \subseteq L$ are left ideals of a semigroup $S$ such that $a L \subseteq K$ for all $a \in S$. If $S$ is $L$-right-reductive, then $S$ can be anti-embedded into $T(L, K)$.

PROOF. Let $\lambda: S \rightarrow T(L), a \rightarrow \lambda_{a}$, where $\lambda_{a}: L \rightarrow L, x \rightarrow a x$, for each $a \in S$. Clearly, $\lambda$ is well defined (since $a L \subseteq L$ for each $a \in S$ ) and $(a b) \lambda=(b \lambda)(a \lambda)$ for all $a, b \in S$. Also, if $\lambda_{a}=\lambda_{b}$, then $a x=b x$ for all $x \in L$ and so $a=b$ by supposition. In addition, $\operatorname{ran} \lambda_{a}=a L \subseteq K$, so each $\lambda_{a} \in T(L, K)$.

The dual of the above result embeds certain semigroups into $T(X, Y)$ for some sets $X$ and $Y$ and, for interest, we now state it explicitly. However, we note that if $1<|Y|$ and $Y \subsetneq X$, then $T(X, Y)$ is not $\mathfrak{S}$-left-reductive for any nonempty subset $\mathfrak{S}$ of $T$; that is, there exist distinct $\alpha, \beta \in T(X, Y)$ such that $\gamma \alpha=\gamma \beta$ for every $\gamma \in \mathfrak{S}$. To see this, choose $x_{1} \in X \backslash Y$ and distinct $y_{1}, y_{2} \in Y$, and let $\alpha, \beta \in T(X)$ be such that $x_{1} \alpha=y_{1}, x_{1} \beta=y_{2}$, and $x \alpha=y_{1}=x \beta$ for every $x \in X \backslash\left\{x_{1}\right\}$. Clearly, $\alpha, \beta$ are distinct elements of $T(X, Y)$ and, since $\alpha|Y=\beta| Y$, we have $\gamma \alpha=\gamma \beta$ for every $\gamma \in \mathfrak{S}$.

THEOREM 17. Suppose that $K \subseteq R$ are right ideals of a semigroup $S$ such that $R a \subseteq K$ for all $a \in S$. If $S$ is $R$-left-reductive, then $S$ can be embedded into $T(R, K)$.

EXAMPLE 18. We give one example of a semigroup which satisfies the algebraic conditions of Theorem 16 but differs from every $T(X, Y)$ with $|Y| \geq 2$. Suppose that $X=\{a, b, c, d\}$, and let $a_{b}$ denote the partial transformation with domain $\{a\}$ and range $\{b\}$. Also let $I_{2}=\{\alpha \in I(X): r(\alpha)<2\}$ : that is, the smallest nonzero ideal of $I(X)$, the symmetric inverse semigroup on $X[1$, Vol. 1, p. 29]. Now write

$$
K=I_{2}, \quad L=K \cup\left\{\left(\begin{array}{ll}
a & b \\
c & d
\end{array}\right)\right\}, \quad S=L \cup\left\{\operatorname{id}_{\{c, d\}}\right\} .
$$

Clearly, $S$ is a semigroup with $\emptyset$ as a zero element, and $S^{2} \neq\{\emptyset\}$ (that is, the operation on $S$ is nontrivial). Also $K \subsetneq L$, and $K, L$ are left ideals of $S$ such that $\alpha L \subseteq K$ for all $\alpha \in S$ (moreover, $\alpha L \neq\{\emptyset\}$ for some $\alpha \in S$ ).

To show that $S$ is $L$-right-reductive, suppose that $a_{b} \gamma=\beta \gamma$ for all $\gamma \in L$. In particular, if $\gamma=b_{a}$ then $a_{b} \cdot b_{a} \neq \emptyset$ implies that $\beta \cdot b_{a} \neq \emptyset$, so $b \in \operatorname{ran} \beta$ and such $\beta \in$ $S$ cannot have rank two; hence, by comparing domains, we see that $\beta=a_{b}$, as required. Also, if $a_{c} \gamma=\beta \gamma$ for all $\gamma \in L$, then $c \in \operatorname{ran} \beta$ and $a \in \operatorname{dom} \beta$; and, if $r(\beta)=2$ then $\beta d_{d} \neq \emptyset$ for $d_{d} \in L$, whereas $a_{c} \cdot d_{d}=\emptyset$. Thus $\beta=a_{c}$, as required. Likewise, if $b_{b} \gamma=\beta \gamma$ for all $\gamma \in L$, then $b_{b} \cdot b_{a} \neq \emptyset$, so $b \in \operatorname{ran} \beta$ and we deduce that $\beta=b_{b}$. Similarly, if $\left(\begin{array}{ll}a & b \\ c & d\end{array}\right) \gamma=\beta \gamma$ for all $\gamma \in L$, then $c, d \in \operatorname{ran} \beta$ and $a, b \in \operatorname{dom} \beta$, and thus $\beta$ must equal $\left(\begin{array}{ll}a & b \\ c & d\end{array}\right)$. Similarly, we can show that if $\alpha, \beta \neq \emptyset$ in $S$ and $\alpha \gamma=\beta \gamma$ for 
all $\gamma \in L$, then $\alpha=\beta$. In addition, it is obvious that $\emptyset \gamma=\beta \gamma$ for all $\gamma \in L$ precisely when $\beta=\emptyset$. Finally, recall that $T(X, Y)$ does not contain a zero if $|Y| \geq 2$.

\section{Acknowledgement}

The authors thank the referee for some very helpful comments.

\section{References}

[1] A. H. Clifford and G. B. Preston, The Algebraic Theory of Semigroups, Mathematical Surveys, No. 7, Vols. 1 and 2 (American Mathematical Society, Providence, RI, 1961 and 1967).

[2] J. M. Howie, An Introduction to Semigroup Theory (Academic Press, London, 1976).

[3] S. Mendes-Gonçalves and R. P. Sullivan, 'Semigroups of transformations restricted by an equivalence', Cent. Eur. J. Math. 8(6) (2010), 1120-1131.

[4] S. Nenthein, P. Youngkhong and Y. Kemprasit, 'Regular elements of some transformation semigroups', Pure Math. Appl. 16(3) (2005), 307-314.

[5] J. Sanwong, B. Singha and R. P. Sullivan, 'Maximal and minimal congruences on some semigroups', Acta Math. Sin. (Engl. Ser.) 25(3) (2009), 455-466.

[6] J. Sanwong and W. Sommanee, 'Regularity and Green's relations on a semigroup of transformations with restricted range', Int. J. Math. Math. Sci. (2008), 11 pp, Art. ID 794013.

[7] R. P. Sullivan, 'Semigroups of linear transformations with restricted kernel', (submitted).

[8] R. P. Sullivan, 'Semigroups of linear transformations with restricted range', Bull. Aust. Math. Soc. 77(3) (2008), 441-453.

[9] J. S. V. Symons, 'Some results concerning a transformation semigroup', J. Aust. Math. Soc. Ser. A 19(4) (1975), 413-425.

SUZANA MENDES-GONÇALVES, Centro de Matemática, Universidade do Minho, 4710 Braga, Portugal

e-mail: smendes@math.uminho.pt

R. P. SULLIVAN, School of Mathematics and Statistics, University of Western Australia, Nedlands, 6009, Australia

e-mail: bob@maths.uwa.edu.au 